\title{
Evaluation of Pectoral Nerve Block in Modified Radical Mastectomy: Comparison of Three Concentrations of Ropivacaine
}

This article was published in the following Dove Press journal:

Clinical Interventions in Aging

\author{
Wei Deng $\mathbb{D}^{1, *}$ \\ $\mathrm{Dan} \mathrm{Fu}^{2, *}$ \\ Liang $\mathrm{He}\left(\mathbb{D}^{3}\right.$
}

'Department of Anesthesiology, The First Hospital of Jiaxing (Affiliated Hospital of Jiaxing University), Jiaxing, People's Republic of China; ${ }^{2}$ Department of Paediatrics, Affiliated Hospital of Guilin Medical University, Guilin, People's Republic of China; ${ }^{3}$ Department of Anesthesiology, Affiliated Hospital of Guilin Medical University, Guilin, People's Republic of China

*These authors contributed equally to this work
Correspondence: Liang He Department of Anesthesiology, Affiliated Hospital of Guilin Medical University, Guilin, People's Republic of China Email jimyliang@hotmail.com
Background: Pectoral nerve block type I (PECS I Block) and type II (PECS II Block) with ropivacaine are relatively new analgesic methods for breast-cancer surgery. We evaluated the safety and efficacy of different concentrations of ropivacaine given in the same volume for the PECS II Block in patients undergoing modified radical mastectomy (MRM).

Patients and Methods: One hundred and twenty women undergoing elective MRM who met inclusion criteria were divided randomly into four groups of 30: control group without PECS II Block and $\mathrm{R}_{0.2 \%}, \mathrm{R}_{0.3 \%}$, and $\mathrm{R}_{0.4 \%}$ groups, who received general anesthesia plus the PECS II Block with ropivacaine at $0.2 \%, 0.3 \%$, and $0.4 \%$, respectively, in a volume of $40 \mathrm{~mL}$.

Results: The postoperative numerical rating scale (NRS) pain score at rest and active was significantly higher in the control group than that in the three ropivacaine groups $(\mathrm{P}<0.05$ for all), and the postoperative NRS score in the $R_{0.3 \%}$ group and $R_{0.4 \%}$ group at 12,24 , and 48 $h$ postoperatively were significantly lower than that in the $R_{0.2 \%}$ group $(\mathrm{P}<0.05$ for all); there was no significant difference between the $\mathrm{R}_{0.3 \%}$ group and $\mathrm{R}_{0.4 \%}$ group. The time when pain was first felt after MRM, the total number of complaints during 3, 6, 12, 24, and $48 \mathrm{~h}$ after MRM, and the total analgesic requirement (tramadol consumption) during the first 24 $h$ postoperatively in the $R_{0.3 \%}$ group and $R_{0.4 \%}$ group were significantly lower than those in the control group and $\mathrm{R}_{0.2 \%}$ group ( $\mathrm{P}<0.05$ for all); there was no significant difference between the $\mathrm{R}_{0.3 \%}$ group and $\mathrm{R}_{0.4 \%}$ group.

Conclusion: A dose of $0.3 \%$ ropivacaine was the optimal concentration for a PECS II Block for patients undergoing MRM because it provided efficacious analgesia during and $>48$ $\mathrm{h}$ after MRM. Increasing the ropivacaine concentration did not improve the analgesia of the PECS II Block significantly.

Keywords: modified radical mastectomy, pectoral nerve block, postoperative analgesia

\section{Introduction}

Breast cancer $(\mathrm{BC})$ is the most common cancer in women worldwide. ${ }^{1,2}$ Mastectomy is indicated for clinical stage-I and -II BC with tumors not involving the chest-muscle fascia. In Beijing, China, 63.97\% of $\mathrm{BC}$ patients underwent modified radical mastectomy from 2006 to $2015 .^{3}$

Modified radical mastectomy (MRM) involves removal of the whole breast: the skin, areola, nipple, and most axillary lymph nodes. During mastectomy, some of the nerves in the chest are severed. Most women have some level of pain in the days after the procedure. Severe acute postoperative pain following breast surgery not only increases the risk of persistent pain and affects recovery, it also leads to longer 
hospitalization and increased healthcare costs. ${ }^{4-6}$ Recent studies have shown that a pectoral nerve block can provide a good analgesic effect for pain after BC surgery. ${ }^{7,8}$

Ultrasound-guided interfacial plane blocks, including pectoral nerve block type I ("PECS I Block") and type II ("PECS II Block"), are novel approaches for blocking the pectoral nerves, long thoracic nerves, and intercostal nerves in the third-to-sixth intercostals, as introduced first by Blanco and colleagues in 2011. ${ }^{9}$ Unlike paravertebral, epidural and thoracic paravertebral blocks, PECS Blocks do not lead to sympathetic blockade, hypotension, pneumothorax, or spinal-cord trauma., ${ }^{6,10}$

Little is known about the effects of different concentrations of ropivacaine in the PECS II Block after MRM. In the present study, our main objective was to compare the efficacy of $0.2 \%, 0.3 \%$, and $0.4 \%$ ropivacaine in an ultrasound-guided PEC Block as analgesia during a 48-h period after MRM. Our secondary aim was to investigate whether the PEC Block reduced the amount of anesthetics used intraoperatively.

\section{Patients and Methods}

\section{Ethical Approval of the Study Protocol}

The study protocol was approved by the ethics committee of the Affiliated Hospital of Guilin Medical University (Guilin, China) and registered in the Chinese Clinical Trial Register (ChiCTR-1800017955). The clinical trial was registered before patient enrollment. The principal investigator was Wei Deng, and the date of registration was 24 August 2018. All participants provided written informed consent before study enrollment. This trial was conducted in accordance with the Declaration of Helsinki.

\section{Participants}

From September 1, 2018 to February 1, 2019, 132 middleaged and older women with $\mathrm{BC}$ who were scheduled for MRM (without immediate reconstruction or tissue expansion) in the Affiliated Hospital of Guilin Medical University were enrolled in the present study. The inclusion criteria were an American Society of Anesthesiologists score of I or II and age between 35 years and 73 years. The exclusion criteria were a bleeding disorder, coagulation abnormality, skin infection, spine/chest deformity, pregnancy or allergy to medication.

Patients were assigned to four groups: control, $0.2 \%$ ropivacaine $\left(\mathrm{R}_{0.2 \%}\right)$, $0.3 \%$ ropivacaine $\left(\mathrm{R}_{0.3 \%}\right)$ or $0.4 \%$ ropivacaine $\left(\mathrm{R}_{0.4 \%}\right)$. Patients in the control group were treated with general anesthesia without the PECS II Block. Patients in the $\mathrm{R}_{0.2 \%}, \mathrm{R}_{0.3 \%}$, and $\mathrm{R}_{0.4 \%}$ groups received general anesthesia plus the PECS II Block. Eligible participants were allocated randomly to four groups according to computer-generated random numbers. An opaque envelope with the codes for group assignments was handed to the consultant anesthetist, who then undertook the nerve block according to the group assignment.

\section{General Anesthesia and PECS II Block}

After patients had been taken to the preoperative area, measurements (pulse oximetry, electrocardiography (ECG), blood pressure) were carried out. Anesthetic management followed a standard protocol. Induction of anesthesia was started with pre-oxygenation for $3 \mathrm{~min}$ and intravenous injection of midazolam ( $0.05 \mathrm{mg} / \mathrm{kg}$ bodyweight), sufentanil $(0.5 \mu \mathrm{g} / \mathrm{kg})$, propofol $(2 \mathrm{mg} / \mathrm{kg})$, and cisatracurium $(0.15 \mathrm{mg} / \mathrm{kg})$. A laryngeal mask was used to complete the airway. Ventilation was set with positive pressure to maintain an end-tidal level of carbon dioxide $\sim 35 \mathrm{mmHg}$.

After the induction of general anesthesia, the patient was placed supine facing towards the contralateral side. She was kept supine with the ipsilateral upper limb in the abducted position, and the midaxillary line and chest were disinfected. A high-frequency linear ultrasound probe (M-Turbo; SonoSite, Bothell, WA, USA) was placed below the lateral third of the clavicle for identification of the pectoralis major and pectoralis minor muscles. First, the linear ultrasound probe was placed cephalocaudally in the infraclavicular region and then moved laterally to locate the axillary vessels directly above the first rib. A 90-mm needle (UniPlex NanoLine; Pajunk, Geisingen, Germany) was inserted directly into the fascial plane between the pectoralis major and pectoralis minor muscles. Then, $20 \mathrm{~mL}$ of ropivacaine was injected (the local anesthetic was injected incrementally with intermittent aspiration of blood). The ultrasound probe was placed laterally in an oblique manner towards the axilla for identification of the serratus anterior muscle (which presents at the level of the third rib). The needle was reinserted into the fascial plane between the pectoralis minor muscle and serratus anterior muscle, and another $20 \mathrm{~mL}$ of ropivacaine was injected. ${ }^{7,11}$

Anesthesia was maintained by an independent anesthesiologist blinded to the allocations of patient groups. We employed $2 \%$ sevoflurane with $50 \%$ oxygen, remifentanil $(0.1-1 \mu \mathrm{g} / \mathrm{kg} . \mathrm{min})$ and propofol $(50-150 \mu \mathrm{g} / \mathrm{kg}$.min) to maintain anesthesia. In addition, cisatracurium $(0.15 \mathrm{mg} / \mathrm{kg})$ was administered according to the surgical protocol. The dose of anesthetics was adjusted to maintain the blood pressure within 
$\pm 20 \%$ of the baseline value. An additional amount of remifentanil (0.1-1.0 $\mu \mathrm{g} / \mathrm{kg}$.min) was injected (i.v.) as needed. If the blood pressure decreased by $>20 \%$ from the baseline value, $250 \mathrm{~mL}$ of $0.9 \%$ (physiologic) saline and ephedrine $(0.1 \mathrm{mg} / \mathrm{kg})$ were given. If the heart rate decreased by $>20 \%$ of the baseline value, atropine $(0.01 \mathrm{mg} / \mathrm{kg})$ was given. At the end of MRM, the effect of cisatracurium was reversed by neostigmine $(0.05 \mathrm{mg} / \mathrm{kg})$ and atropine $(0.02 \mathrm{mg} / \mathrm{kg})$.

Heart rate, systolic blood pressure, diastolic blood pressure, oxygen saturation, and end-tidal carbon dioxide were recorded before the start of MRM, upon skin incision, and every 5 min during MRM. The duration of the surgical procedure and anesthesia, recovery time (i.e., the time from waking to leaving the resuscitation room), amount of fluid in and out, as well as the type and dose of opioids used were also recorded.

\section{Pain Assessment}

Pain was assessed according to a standard numeric rating scale (NRS) with 0 indicating "no pain" and 10 indicating "extreme pain". Patients were asked to place a marker along the linear scale to indicate their level of pain.

Rescue analgesia was provided by nursing staff who were blinded to the study protocol when the patient complained of pain and having a NRS score $>3$ at rest. Tramadol $(2 \mathrm{mg} / \mathrm{kg}$, i.v. $)$ was the rescue analgesic in all groups. A maximum of four doses were given in $24 \mathrm{~h}$. The total analgesic requirement (tramadol consumption) during the first $24 \mathrm{~h}$ after surgery was recorded.

For each patient, the NRS score 3, 6, 12, 24 and 48 $\mathrm{h}$ after MRM, as well as the prevalence of nausea, vomiting, and limb paresthesia, were recorded while at rest (i.e., immobile in bed) and active (i.e., moving in or out of bed). Patient satisfaction was assessed and recorded $48 \mathrm{~h}$ after surgery. Parameters were observed and recorded by another researcher who was not informed of group allocations.

\section{Primary Outcomes and Secondary Outcomes}

The primary outcomes were the NRS score 3, 6, 12, 24 and $48 \mathrm{~h}$ after surgery, time that pain was first felt after MRM, the total analgesic requirement (tramadol consumption) during the first $24 \mathrm{~h}$, and total number of complaints during 3, 6 , 12, 24 and $48 \mathrm{~h}$. The secondary outcomes were the dose of remifentanil and propofol administered and the satisfaction score of patients (1-10, whereby 10 is the highest).

\section{Statistical Analyses}

The sample size was based on a preliminary experiment in which the NRS score for pain at rest $24 \mathrm{~h}$ after surgery in the $\mathrm{R}_{0.2 \%}$ group ( $\mathrm{n}=10$ patients) was $2.50 \pm 0.78$, whereas the NRS score in the $\mathrm{R}_{0.4 \%}$ group ( $\mathrm{n}=10$ patients) was $1.23 \pm 0.43$. The test level $\alpha$ was taken as 0.05 , with $Z_{0.05 / 2}=1.96$. The power level $1-\beta$ was taken as 0.8 , with $Z_{0.2}=0.84$. Therefore, a sample size of 30 was required in each group. Assuming that $10 \%$ of patients would drop out, 33 cases were recruited for each group.

Data were evaluated by an independent analyst blinded to the allocations of patient groups. Statistical analyses were done using SPSS v25.0 (IBM, Armonk, NY, USA). Continuous variables are expressed as the mean $\pm \mathrm{SD}$. Differences were assessed using one-way analysis of variance (ANOVA). The prevalence of nausea, vomiting, and limb paresthesia is presented as a percentage, and differences among groups were evaluated using the chi-square test. Pairwise comparison of one-way ANOVA was made using post hoc analysis and the Student-Newman-Keuls $Q$-test. The corrected P-value was obtained directly, and the cutoff value was 0.05 .

\section{Results}

One hundred and thirty-two patients were screened for eligibility for inclusion in the present study, and the consort diagram is shown in Figure 1. Ultimately, 120 women were included in the final analysis. The demographic features of patients in the four treatment groups are described in Table 1.

$\mathrm{R}_{0.2 \%}, \mathrm{R}_{0.3 \%}$ and $\mathrm{R}_{0.4 \%}$ groups showed significant differences in the dose of propofol and remifentanil used compared with that in the control group $(\mathrm{P}<0.05$ for all comparisons). $\mathrm{R}_{0.3 \%}$ and $\mathrm{R}_{0.4 \%}$ groups showed significant differences in the dose of propofol and remifentanil used compared with that in the $\mathrm{R}_{0.2 \%}$ group $(\mathrm{P}<0.05$ for all comparisons), No significant differences in the duration of the surgical procedure $(\mathrm{P}=0.995)$ or duration of anesthesia $(\mathrm{P}=0.517)$ were observed among the four groups, and

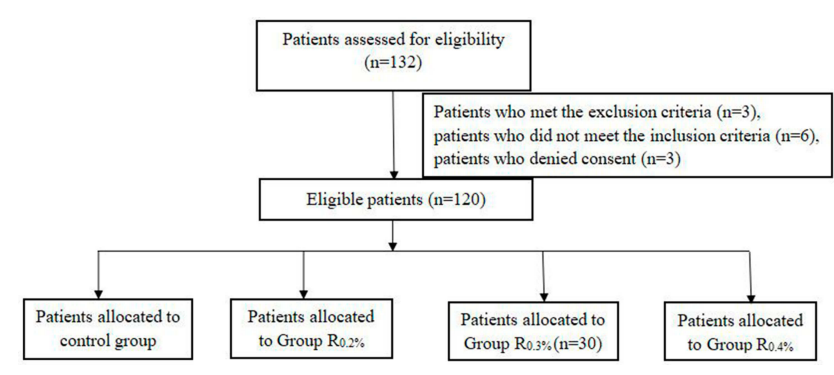

Figure I Consort diagram for the study. 
Table I Basic Characteristics of Patients in the Four Groups ( $n=30$ for Each Group)

\begin{tabular}{|l|l|l|l|l|l|}
\hline & Control & $\mathbf{R}_{\mathbf{0 . 2}}$ & $\mathbf{R}_{\mathbf{0 . 3}}$ & $\mathbf{R}_{\mathbf{0 . 4}}$ & $\mathbf{P}$ \\
\hline Age (years) & $47.5 \pm 10.0$ & $49.4 \pm 12.0$ & $46.3 \pm 9.1$ & $52.6 \pm 9.7$ & 0.444 \\
Height (cm) & $155.4 \pm 4.2$ & $155.1 \pm 3.2$ & $154.6 \pm 3.6$ & $154.4 \pm 3.8$ & 0.741 \\
Weight (kg) & $56.6 \pm 8.6$ & $56.0 \pm 5.8$ & $55.2 \pm 7.4$ & $55.7 \pm 7.2$ & 0.906 \\
Procedure duration (min) & $141.4 \pm 31.2$ & $139.7 \pm 35.3$ & $138.8 \pm 42.0$ & $139.7 \pm 48.9$ & 0.995 \\
Duration of anesthesia (min) & $178.8 \pm 58.7$ & $161.1 \pm 34.0$ & $165.7 \pm 43.7$ & $163.9 \pm 56.2$ & 0.517 \\
ASA class I (n/\%) & $12(40 \%)$ & $13(43.3 \%)$ & $14(46.7 \%)$ & $15(50 \%)$ & - \\
ASA class II (n/\%) & $18(60 \%)$ & $17(56.7 \%)$ & $16(53.3 \%)$ & $15(50 \%)$ \\
\hline
\end{tabular}

Notes: Statistical tests: Pairwise comparisons of groups analyzed by one-way ANOVA were made using post hoc analyses and the Student-Newman-Keuls $Q$-test.

Table 2 Analgesic Use After Surgery and Recovery Time in the Four Groups ( $\mathrm{n}=30$ in Each Group)

\begin{tabular}{|l|l|l|l|l|l|l|}
\hline & Control & $\mathbf{R}_{\mathbf{0 . 2}}$ & $\mathbf{R}_{\mathbf{0 . 3}}$ & $\mathbf{R}_{\mathbf{0 . 4}}$ & $\chi^{\mathbf{2}}$ \\
\hline Remifentanil $(\mu \mathrm{g})$ & $1371.3 \pm 490.2$ & $445.7 \pm 120.9$ & $244.7 \pm 95.4$ & $235.3 \pm 94.4$ & 127.978 & 0.000 \\
Propofol (mg) & $673.7 \pm 209.4$ & $479.0 \pm 78.4$ & $342.0 \pm 82.1$ & $323.7 \pm 96.7$ & 45.453 & 0.000 \\
Recovery time (min) & $31.37 \pm 3.01$ & $22.50 \pm 0.94$ & $21.53 \pm 1.36$ & $21.03 \pm 1.24$ & 209.606 & 0.000 \\
\hline
\end{tabular}

Notes: Statistical tests: Pairwise comparisons of groups analyzed by one-way ANOVA were made using post hoc analyses and the Student-Newman-Keuls $Q$-test as well as the chi-square test.

there was no significant difference between the $\mathrm{R}_{0.3 \%}$ group and $\mathrm{R}_{0.4 \%}$ group. The recovery time in all three ropivacaine groups was $\sim 10$-min shorter than that in the control group ( $\mathrm{P}<0.05$ for all comparisons). The recovery time in the $\mathrm{R}_{0.3 \%}$ group and $\mathrm{R}_{0.4 \%}$ group differed significantly from that in the $\mathrm{R}_{0.2 \%}$ group $(\mathrm{P}<0.05$ for all comparisons) but there was no significant difference between the $\mathrm{R}_{0.3 \%}$ group and $\mathrm{R}_{0.4 \%}$ group (Table 2 ).

The NRS score recorded at different times after surgery in the four groups is presented in Figure 2. The postoperative NRS score in the $\mathrm{R}_{0.2 \%}$ group, $\mathrm{R}_{0.3 \%}$ group and $\mathrm{R}_{0.4 \%}$ group 12 , 24 , and $48 \mathrm{~h}$ after surgery when patients were at rest or active was significantly lower than that in the control group $(\mathrm{P}<0.05$ for all comparisons). The NRS score in the $\mathrm{R}_{0.3 \%}$ group and $\mathrm{R}_{0.4 \%}$ group 12,24 , and $48 \mathrm{~h}$ after surgery when patients were at rest or active was significantly lower than that in the $\mathrm{R}_{0.2 \%}$ group ( $\mathrm{P}<0.05$ for all comparisons). The postoperative NRS score did not differ significantly between the $\mathrm{R}_{0.3 \%}$ group and $\mathrm{R}_{0.4 \%}$ group.

The time when pain was first felt after MRM and total number of complaints during 3, 6, 12, 24 and $48 \mathrm{~h}$ in the three ropivacaine groups were lower than those in the control group and $\mathrm{R}_{0.2 \%}$ group ( $\mathrm{P}<0.05$ for all comparisons) and there was no significant difference between the $\mathrm{R}_{0.3 \%}$ group and $\mathrm{R}_{0.4 \%}$ group. The total analgesic requirement (tramadol consumption) in the $\mathrm{R}_{0.3 \%}$ group and $\mathrm{R}_{0.4 \%}$ group within 24 $\mathrm{h}$ after surgery was significantly lower than that in the control group and $\mathrm{R}_{0.2 \%}$ group ( $\mathrm{P}<0.05$ for all comparisons) (Figure 3), and there was no significant difference between the $\mathrm{R}_{0.3 \%}$ group and $\mathrm{R}_{0.4 \%}$ group. Compared with that in the control group, the satisfaction score in the ropivacaine groups $48 \mathrm{~h}$ after surgery was greater by $>30 \%(\mathrm{P}<0.05$
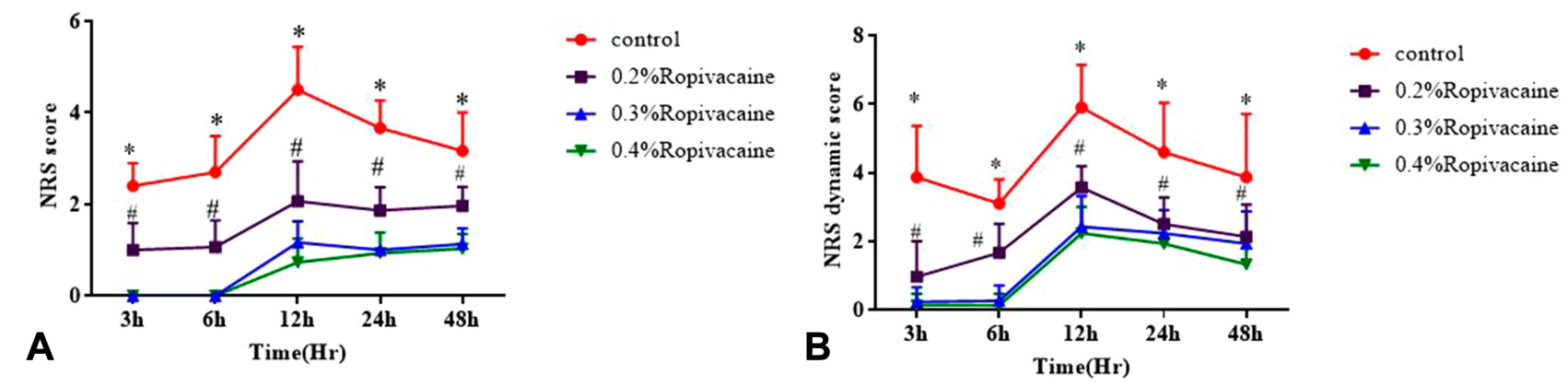

Figure 2 NRS score at different times after surgery in the four groups. (A) NRS score when patients were at rest. (B) NRS score when patients were active. *P<0.05 compared with $\mathrm{R}_{0.2 \%}, \mathrm{R}_{0.3 \%}$ and $\mathrm{R}_{0.4 \%}$ groups, ${ }^{\#} \mathrm{P}<0.05$ compared with $\mathrm{R}_{0.3 \%}$ and $\mathrm{R}_{0.4 \%}$ groups. 

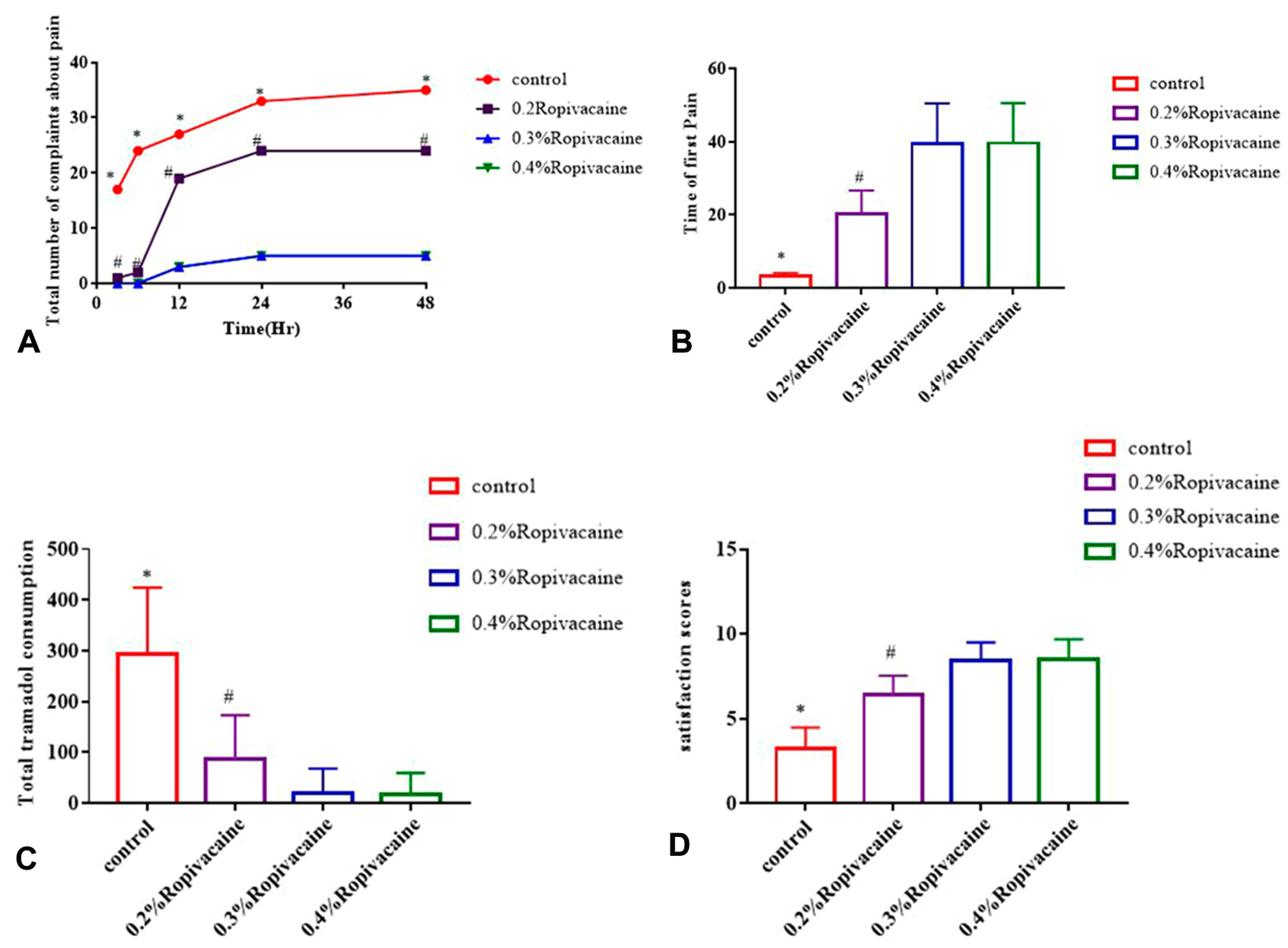

Figure 3 (A) Total number of complaints during 3, 6, 12, 24 and 48 h. (B) Time that pain was first felt after surgery. (C) Total tramadol consumption (mg) in 24 h. (D) Satisfaction score in the ropivacaine groups $48 \mathrm{~h}$ after surgery. ${ }^{*} \mathrm{P}<0.05$ compared with $\mathrm{R}_{0.2 \%}, \mathrm{R}_{0.3 \%}$ and $\mathrm{R}_{0.4 \%}$ groups, ${ }^{\#} \mathrm{P}<0.05$ compared with $\mathrm{R}_{0.3 \%}$ and $\mathrm{R}_{0.4 \%}$ groups.

for all comparisons) (Figure 3). Moreover, compared with that in the $\mathrm{R}_{0.2 \%}$ group, the postoperative satisfaction score in the $\mathrm{R}_{0.3 \%}$ and $\mathrm{R}_{0.4 \%}$ groups was $>20 \%$ better (Figure 3 ).

The prevalence of postoperative nausea in the $\mathrm{R}_{0.2 \%}, \mathrm{R}_{0.3 \%}$ and $\mathrm{R}_{0.4 \%}$ groups was lower than that in the control group $(\mathrm{P}<$ 0.05 for all comparisons), but the prevalence of vomiting did not differ significantly among the four groups $(\mathrm{P}=0.128)$ (Table 3). None of the patients reported limb paresthesia.

\section{Discussion}

This prospective, double-blinded, randomized study was carried out to find the appropriate concentration of ropivacaine for the PECS II Block. We found that $40 \mathrm{~mL}$ of $0.3 \%$ ropivacaine reduced pain and increased patient satisfaction. The highest concentration of ropivacaine $(0.4 \%)$ did not provide a significant advantage with regard to postoperative analgesia using the PECS II Block.

The postoperative NRS score in the $\mathrm{R}_{0.3 \%}$ group and $\mathrm{R}_{0.4 \%}$ group was significantly lower than that in the $\mathrm{R}_{0.2 \%}$ group. We cannot state that the difference in pain was clinically relevant using only the NRS score because the difference in the NRS score was only 2 points. Nevertheless, compared with the $\mathrm{R}_{0.2 \%}$ group, the $\mathrm{R}_{0.3 \%}$ group showed values of $94.05 \%, 81.4 \%$, and $75.77 \%$ for

Table 3 Prevalence of Adverse Events in the Four Groups ( $n=30$ in Each Group)

\begin{tabular}{|l|l|l|l|l|l|l|}
\hline & Control & $\mathbf{R}_{\mathbf{0 . 2}}$ & $\mathbf{R}_{\mathbf{0 . 3}}$ & $\mathbf{R}_{\mathbf{0 . 4}}$ & $\chi^{\mathbf{2}}$ \\
\hline Nausea (n/\%) & II (36.67\%) & $4(13.33 \%)$ & $3(10 \%)$ & $4(13.33 \%)$ & 9.128 \\
Vomiting (n/\%) & $8(26.67 \%)$ & $4(13.33 \%)$ & $2(6.67 \%)$ & $3(10 \%)$ & 0.028 \\
Limb paresthesia (n/\%) & 0 & 0 & 0 & 0 & 5.688 & 0.128 \\
\hline
\end{tabular}

Note: Statistical test: Chi-square test. 
the time when pain was first felt after MRM, the total number of complaints, and total analgesic requirement (tramadol consumption), respectively. The $\mathrm{R}_{0.4 \%}$ group showed values of $94.64 \%, 81.4 \%$, and $78.96 \%$, for the time pain was first felt after MRM, the total number of complaints, and total analgesic requirement (tramadol consumption), respectively. The $\mathrm{R}_{0.3 \%}$ group did not show a significant difference for the prevalence of the time when pain was first felt after MRM, the total number of complaints, or total analgesic requirement (tramadol consumption) compared with that in the $\mathrm{R}_{0.4 \%}$ group. These data suggested a true clinical difference in pain between the patient groups undergoing the PECS II Block. Therefore, from a clinical viewpoint, $0.3 \%$ ropivacaine may provide efficacious analgesia for patients during and after MRM.

Pectoral nerve blocks have been shown to provide efficacious analgesia after breast surgery. In the PECS I Block, anesthetic is injected only into the fascial plane between the pectoralis major and pectoralis minor muscles to block the medial and lateral pectoral nerves that innervate the pectoralis muscles. In the PECS II Block, an additional injection of local anesthetic is administered in the plane between the pectoralis minor and serratus anterior muscles. The aims of the PECS II Block are blockade of the lateral and median pectoral nerves at an inter-fascial plane between the pectoralis major and pectoralis minor muscles, long thoracic nerve, thoracic intercostal nerves from T2 to T6, and thoracodorsal nerve. An ultrasoundguided PECS II Block is safer, easier and faster to operate, and has longer analgesia than a paravertebral nerve block or epidural nerve block in MRM for BC. ${ }^{12-15}$ The PECS II Block guided by ultrasound applied to MRM can not only reduce intraoperative use of opioids, it has less of an effect on hemodynamics. Thus, for patients with hypertension and coronary heart disease undergoing BC surgery, this strategy greatly reduces the risk of anesthesia, risk of postoperative complications, and improves the postoperative quality of life of patients. However, whether this strategy can lower the prevalence of persistent pain after BC treatment is not known. Recent studies have shown that the PECS II Block can prevent chronic pain 3 months after breast surgery. ${ }^{16}$ Few scholars have focused on application of the PECS II Block and its effect on persistent pain after BC treatment.

Our study demonstrated that reduction of pain after MRM through a PECS II Block with ropivacaine was dependent upon the ropivacaine concentration, and that $0.2 \%$ ropivacaine could not offer efficacious analgesia for MRM. Forty milliliters of ropivacaine was used to cover the entire pectoral nerve block. Studies have shown that $40 \mathrm{~mL}$ of ropivacaine is safe for nerve blockade, ${ }^{17}$ and we found no adverse reactions using this volume.

However, when the ropivacaine concentration was increased to $0.3 \%$ and $0.4 \%$, the postoperative NRS score 12,24 , and $48 \mathrm{~h}$ after MRM when patients were at rest or active was significantly lower than that in the $\mathrm{R}_{0.2 \%}$ group. Yamada et $\mathrm{al}^{18}$ and Taha et $\mathrm{al}^{19}$ demonstrated that ropivacaine had a concentration-dependent analgesic effect. Yinglan and colleagues showed that increasing the ropivacaine concentration under the same volume in ultrasound-guided regional anesthesia resulted in a progressive increase in the analgesic effect, ${ }^{20}$ but $0.4 \%$ ropivacaine did not show a better analgesic effect than $0.3 \%$ ropivacaine. Increasing the plasma concentration of ropivacaine may increase the risk of a toxic reaction. Research has shown that, in a transverse facial nerve block, in terms of efficacy and safety, use of $0.375 \%$ ropivacaine is preferred and improves the degree of satisfaction $24 \mathrm{~h}$ after surgery. The findings stated above are similar to our observations. The safe and efficacious concentration of ropivacaine used in a reverse facial nerve block by Sun and colleagues is close to the one that we used. ${ }^{21}$ Another study found that, in the supraclavicular brachial plexus block, ultrasoundguided double-injection of ropivacaine $(0.257 \% \mathrm{w} / \mathrm{v}$; $40 \mathrm{~mL}$ ) could provide successful blockade of the brachial plexus in $90 \%$ of middle-aged adults with normal body habitus. ${ }^{17}$ Thus, $0.3 \%$ ropivacaine may be better than $0.4 \%$ ropivacaine when used in patients undergoing MRM.

Some studies have shown that visual analog scale (VAS) pain scores at rest and while active during the first $24 \mathrm{~h}$ after surgery reveal obvious pain, and that the heart rate and blood pressure are increased significantly. ${ }^{22-24} \mathrm{We}$ found that the peak of pain after MRM occurred from 24 $\mathrm{h}$ to $48 \mathrm{~h}$. Notably, pectoral nerve blockade has been found to be easy to carry out under ultrasound guidance, ${ }^{25-27}$ and that the only complication is hematoma. ${ }^{15,28}$

Our study had three main limitations. First, we did not give a sham block to the control group because of the ethical considerations of giving an injection without administering a therapeutic drug. Second, we adjusted the demand for preoperative remifentanil in the experiments, but the Bispectral Index was not measured, which caused a biased in our study. Remifentanil was used in all four groups, and remifentanil is likely to cause opioidinduced hyperalgesia in patients and, in the control 
group, it was likely to increase the demand for pain medication after MRM. Third, the study cohort was only 30 patients; a high-powered study with many more patients is needed to confirm our findings.

\section{Conclusions}

A dose of $0.3 \%$ ropivacaine was found to be the optimal concentration for a PECS II Block for patients undergoing MRM because it provided efficacious analgesia during and $>48 \mathrm{~h}$ after MRM. Increasing the ropivacaine concentration did not improve the analgesia of the PECS II Block significantly.

\section{Data Sharing Statement}

The authors intend to share individual deidentified participant data, and all the data are published in the China Clinical Trial Registration Center. No additional unpublished data are available. You can $\log$ in to the China Clinical Trial Registration Center to share our data. When the article is published, it can be used permanently.

\section{Funding}

This study was supported by Natural Science Foundation of China (81860239), 2019 Jiaxing Key Discipline of Medicine - Anesthesiology (Supporting Subject) (2019zc-06), Guangxi Medical and Health Key Cultivation Discipline Construction Project and Item No. 2020-XB020 .

\section{Disclosure}

Wei Deng and Dan Fu are co-first authors for this study. The authors report no conflicts of interest in this work.

\section{References}

1. Siegel RL, Miller KD, Jemal A. Cancer statistics, 2018. CA Cancer J Clin. 2018;68(1):7-30. doi:10.3322/caac.21442

2. Chen W, Zheng R, Baade PD. Cancer statistics in China, 2015. CA Cancer J Clin. 2016;66(2):115-132. doi:10.3322/caac.21338

3. Bao X, Sun K, Tian X, et al. Present and changing trends in surgical modalities and neoadjuvant chemotherapy administration for female breast cancer in Beijing, China: a 10-year (2006-2015) retrospective hospitalization summary report-based study. Thorac Cancer. 2018;9 (6):707-717. doi:10.1111/1759-7714.12636

4. Hickey OT, Burke SM, Hafeez P, Mudrakouski AL, Hayes ID, Shorten GD. Severity of acute pain after breast surgery is associated with the likelihood of subsequently developing persistent pain. Clin J Pain. 2010;26(7):556-560. doi:10.1097/AJP.0b013e3181dee988

5. Masselin-Dubois A, Attal N, Fletcher D, et al. Are psychological predictors of chronic postsurgical pain dependent on the surgical model? A comparison of total knee arthroplasty and breast surgery for cancer. J Pain. 2013;14(8):854-864. doi:10.1016/j.jpain.2013.02.013
6. Versyck B, van Geffen G-J, Van Houwe P. Prospective double blind randomized placebo-controlled clinical trial of the pectoral nerves (pecs) block type II. J Clin Anesth. 2017;40:46-50. doi:10.1016/j. jclinane.2017.03.054

7. Karaca O, Pinar HU, Arpacı E, Dogan R, Cok OY, Ahiskalioglu A. The efficacy of ultrasound-guided type-I and type-II pectoral nerve blocks for postoperative analgesia after breast augmentation: a prospective, randomised study. Anaesth Crit Care Pain Med. 2019;38(1):47-52. doi:10.1016/j.accpm.2018.03.009

8. Blanco R. The 'pecs block': a novel technique for providing analgesia after breast surgery. Anaesthesia. 2011;66(9):847-848. doi:10.1111/j.1365-2044.2011.06838.x

9. Gaurav K, Geeta C. Ropivacaine: a review of its pharmacology and clinical use. Indian J Anaesth. 2011;55(2):104-110. doi:10.4103/ 0019-5049.79875

10. Versyck B, van Geffen G-J, Chin K-J. Analgesic efficacy of the pecs II block: a systematic review and meta-analysis. Anaesthesia. 2019;74(5):663-673. doi:10.1111/anae.14607

11. Blanco R, Fajardo M, Parras Maldonado T. Ultrasound description of pecs II (modified pecs I): a novel approach to breast surgery. Rev Esp Anestesiol Reanim. 2012;59(9):470-475. doi:10.1016/j.redar.2012.07.003

12. Wahba SS, Sahar MK. Thoracic paravertebral block versus pectoral nerve block for analgesia after breast surgery. Egypt J Anaesth. 2014;30(2):129-135. doi:10.1016/j.egja.2013.10.006

13. Sopena-Zubiria LA, Fernández-Meré LA, Valdés Arias C. Thoracic paravertebral block compared to thoracic paravertebral block plus pectoral nerve block in reconstructive breast surgery. Rev Esp Anestesiol Reanim. 2012;59(1):12-17. doi:10.1016/j.redar.2011.10.001

14. Morioka H, Kamiya Y, Yoshida T, Baba H. Pectoral nerve block combined with general anesthesia for breast cancer surgery: a retrospective comparison. JA Clin Rep. 2015;1:15. doi:10.1186/ s40981-015-0018-1

15. Yalamuri S, Klinger RY, Bullock WM, Glower DD, Bottiger BA, Gadsden JC. Pectoral fascial (PECS) I and II blocks as rescue analgesia in a patient undergoing minimally invasive cardiac surgery. Reg Anesth Pain Med. 2017;42(6):764-776. doi:10.1097/ AAP.0000000000000661

16. De Cassai A, Bonanno C, Sandei L, Finozzi F, Carron M, Marchet A. PECS II block is associated with lower incidence of chronic pain after breast surgery. Korean J Pain. 2019;32(4):286-291. doi:10.3344/kjp.2019.32.4.286

17. Fang G, Wan L, Mei W, Yu HH, Luo AL. The minimum effective concentration (MEC90) of ropivacaine for ultrasound-guided supraclavicular brachial plexus block. Anaesthesia. 2016;71:700-705. doi:10.1111/anae.13445

18. Yamada K, Inomata S, Tanaka M. The ropivacaine concentration required for ultrasound-guided ilioinguinal/iliohypogastric nerve block in pediatric patients. Anesth Analg. 2016;123(1):175-178. doi:10.1213/ANE.0000000000001329

19. Taha AM, Abd-Elmaksoud AM. Ropivacaine in ultrasound-guided femoral nerve block: what is the minimal effective anaesthetic concentration (EC90)? Anaesthesia. 2014;69(7):678-682. doi:10.1111/ anae. 12607

20. Su Y, Zhang Z, Zhang Y, Li H, Wei SH. Efficacy of ropivacaine by the concentration of $0.25 \%, 0.5 \%$, and $0.75 \%$ on surgical performance, postoperative analgesia, and patient's satisfaction in inguinal hernioplasty: a randomized controlled trial. Patient Prefer Adherence. 2015;9:1375-1379. doi:10.2147/PPA.S93276

21. Sun N, Wang S, Ma P, et al. Postoperative analgesia by a transversus abdominis plane block using different concentrations of ropivacaine for abdominal surgery: a meta-analysis. Clin J Pain. 2017;33 (9):853-863. doi:10.1097/AJP.0000000000000468

22. Singh PM, Borle A, Kaur M, Trikha A, Sinha A. Opioid-sparing effects of the thoracic interfascial plane blocks: a meta-analysis of randomized controlled trials. Saudi J Anaesth. 2018;1:103-111. doi:10.4103/sja.SJA_382_17 
23. Goswami S, Kundra P, Bhattacharyya J. Pectoral nerve block 1 versus modified pectoral nerve block 2 for postoperative pain relief in patients undergoing modified radical mastectomy: a randomized clinical trial. Br J Anaesth. 2017;119(4):830-835. doi:10.1093/bja/ aex 201

24. Kumar S, Goel D, Sharma SK, et al. A randomised controlled study of the post-operative analgesic efficacy of ultrasound-guided pectoral nerve block in the first $24 \mathrm{~h}$ after modified radical mastectomy. Indian $J$ Anaesth. 2018;62(6):436-442. doi:10.4103/ija.IJA_523_17

25. Hongxu J, Zhang T, Sun X, Wang Z, Wang F. Efficacy of ultrasound guided pectoral nerve block versus thoracic paravertebral block for postoperative analgesia after radical mastectomy. $\mathrm{Br} J$ Anaesth. 2018;34(2):126-129.
26. Kulhari S, Bharti N, Bala I, Arora S, Singh G. Efficacy of pectoral nerve block versus thoracic paravertebral block for postoperative analgesia after radical mastectomy: a randomized controlled trial. Br J Anaesth. 2016;117(3):382-386. doi:10.1093/bja/aew223

27. Nair AS, Sahoo RK, Ganapathy M, Mudunuri R. Ultrasound guided blocks for surgeries/procedures involving chest wall (pecs 1, 2 and serratus plane block). Anaesth Pain Intens Care. 2015;19:348-351.

28. Bashandy GM, Abbas DN. Pectoral nerves I and II blocks in multimodal analgesia for breast cancer surgery: a randomized clinical trial. Reg Anesth Pain Med. 2015;40:68-74. doi:10.1097/AAP.0000 000000000163

\section{Publish your work in this journal}

Clinical Interventions in Aging is an international, peer-reviewed journal focusing on evidence-based reports on the value or lack thereof of treatments intended to prevent or delay the onset of maladaptive correlates of aging in human beings. This journal is indexed on PubMed Central, MedLine, CAS, Scopus and the Elsevier
Bibliographic databases. The manuscript management system is completely online and includes a very quick and fair peer-review system, which is all easy to use. Visit http://www.dovepress.com/ testimonials.php to read real quotes from published authors. 